\title{
Thermal and fast neutron detection with two CLYC scintillators
}

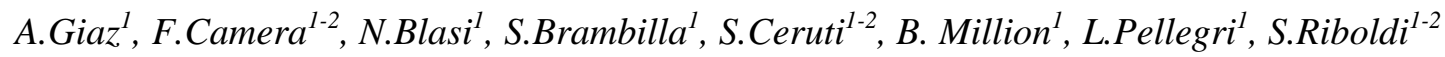 \\ ${ }^{1}$ INFN Sezione di Milano, Milano, Italy \\ ${ }^{2}$ Università degli studi di Milano, Milano, Italy
}

\begin{abstract}
The CLYC scintillator has the capability to identify gamma rays and fast/thermal neutrons and it is characterized by a good properties for the gamma ray detection. In this work, the response to thermal and fast neutrons of two CLYC 1"x1" crystals (one enriched with ${ }^{7} \mathrm{Li}$ and one with ${ }^{6} \mathrm{Li}$ ) was measured. The thermal neutrons were measured using both detectors. The measurements of fast neutrons were performed at Neutron Generator facility (Frascati, Italy) and at CN accelerator of LNL. At the neutron generator facility, neutrons at 2.5 and $14.1 \mathrm{MeV}$ were measured. The response to fast neutrons was measured also from $1.9 \mathrm{MeV}$ up to $3.8 \mathrm{MeV}$ at LNL.
\end{abstract}

\section{Introduction}

The crystal $\mathrm{Cs}_{2} \mathrm{LiYCl}_{6}: \mathrm{Ce}$ (CLYC) it is a very interesting scintillator material because of its excellent energy resolution and its capability to identify gamma rays and fast/thermal neutrons. The crystal $\mathrm{Cs}_{2} \mathrm{LiYCl}_{6}: \mathrm{Ce}$ contains ${ }^{6} \mathrm{Li}$ and ${ }^{35} \mathrm{Cl}$ isotopes, therefore, it is possible to detect thermal neutrons through the reaction ${ }^{6} \mathrm{Li}(\mathrm{n}, \alpha) \mathrm{t}$ (cross section of 940 barns). While ${ }^{35} \mathrm{Cl}$ ions allows to measure fast neutrons through the reactions ${ }^{35} \mathrm{Cl}(\mathrm{n}, \mathrm{p}){ }^{35} \mathrm{~S}$ and ${ }^{35} \mathrm{Cl}(\mathrm{n}, \alpha){ }^{32} \mathrm{P}$ (the cross section is of the order of 0.1 barns) [1-6]. In this work, the response to thermal and fast neutrons of two CLYC 1" 1 1" crystals was measured: the first one, enriched with ${ }^{6} \mathrm{Li}$ at $95 \%$, is ideal for thermal neutron measurements (CLYC-6) while the second one, with an enrichment of ${ }^{7} \mathrm{Li}$ higher than $99 \%$ (CLYC-7), is suitable for fast neutron measurements.

Thermal neutrons from an AmBe source were measured using both detectors, in the gamma spectroscopy laboratory of the University of Milano, Italy. The measurements of fast neutrons were performed at Frascati (Italy) Neutron Generator facility where a deuterium beam was accelerated on a deuterium or on a tritium target, providing neutrons of $2.5 \mathrm{MeV}$ or $14.1 \mathrm{MeV}$, respectively. The response to fast neutrons was measured also at CN accelerator of Laboratori Nazionali di Legnaro (LNL), Italy. At LNL a proton beam was accelerated at $5.5,5$ and $4 \mathrm{MeV}$ on a ${ }^{7} \mathrm{LiF}$ target, to produce neutron with energy from 1.9 up to $3.8 \mathrm{MeV}$. The detector were placed at $0^{\circ}$ or at $90^{\circ}$ from the target to have for each beam energy two different neutrons energies.

The thermal neutron measurements are described in section 2. It is focuses on the differences between CLYC-6 and CLYC-7. Section 3 describes the fast neutron detection. In particular, section 3.1 is dedicated to the measurements performed at Neutron Generator of ENEA Laboratories in Frascati. Section 3.2, instead is dedicated to fast neutron detection exploiting both energy signal and the Time of Flight (TOF) technique at LNL. The conclusions of the work are in section 4.

\section{Thermal Neutron Measurements}

The thermal detection capability arises from ${ }^{6} \mathrm{Li}$ ions, which have a 940 barns cross section for the reaction ${ }^{6} \mathrm{Li}(\mathrm{n}, \alpha)^{3} \mathrm{H}$ [3]. Thermal neutrons were measured with both CLYC-6 and CLYC-7 crystals placed in the same position relative to the AmBe source. 
The only difference between the two crystals is the Li isotope enrichment. In particular, as already mentioned, our CLYC-7 crystal has an enrichment of ${ }^{7} \mathrm{Li}$ higher than $99 \%$ while the CLYC-6 detector has an enrichment of $\sim 95 \%$ of ${ }^{6} \mathrm{Li}$. These two different isotopes produce different sensitivity to thermal neutrons. In figure 1 the comparison between the energy spectra measured with the CLYC- 6 and the CLYC-7 detectors are shown in left and right panel respectively. Both detectors were placed over a 40 $\mathrm{cm}$ box of paraffin with a source of AmBe in the centre. The spectra of figure 6 are normalized on the ${ }^{137} \mathrm{Cs}$ peak. The $3.2 \mathrm{MeV}$ electron equivalent peak, induced by thermal neutrons, clearly visible in CLYC-6 spectrum, is absent in the CLYC-7 one. By estimating the counts in the thermal neutron peak, we can conclude that the CLYC-7 detector has an efficiency to thermal neutrons of $\sim 0.3 \%$ with respect to the CLYC-6 one. Similar results were observed by D'Olympia et al., as shown in Ref. [4].
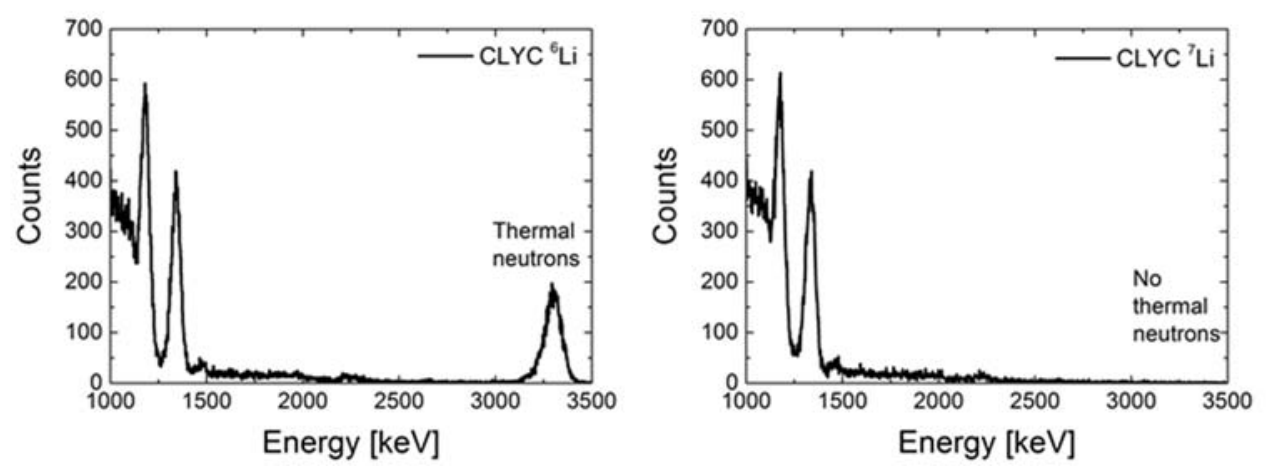

Fig. 1: the energy spectra measured with CLYC-6 (left panel) and CLYC-7 (right panel), respectively. An $\mathrm{AmBe}(\mathrm{Ni})$ source in a paraffin box and a ${ }^{60} \mathrm{Co}$ and a ${ }^{137} \mathrm{Cs}$ were present.

\section{Fast Neutrons Measurements}

Fast neutrons are detected exploiting the reactions ${ }^{35} \mathrm{Cl}(\mathrm{n}, \mathrm{p}){ }^{35} \mathrm{~S}$ and ${ }^{35} \mathrm{Cl}(\mathrm{n}, \alpha){ }^{32} \mathrm{P}$. The energy of the outgoing proton or $\alpha$ particle is linearly related to the neutron energy. For this reason, CLYC scintillators are neutron spectrometers. Furthermore, the neutron kinetic energy can also be measured via the Time of Flight technique (FWHM $<1 \mathrm{~ns}$ ) [5-6].

\subsection{Fast Neutron Measurements at Neutron Generator}

A measurement was performed at the Neutron Generator at ENEA laboratories in Frascati (Italy), which provided a monochromatic beam of $14.1 \mathrm{MeV}$ and $\sim 2.5 \mathrm{MeV}$ neutrons. The two CLYC scintillators were coupled with HAMAMATSU R6233-100Mod photomultiplier tubes (PMT) and with two standard HAMAMATSU E1198-26 and E1198-27 voltage dividers. The signal of both detectors were digitized using Le Croy waverunner HDO 605412 bit oscilloscope.

The 14.1 MeV neutron emission was obtained by bombarding a tritium doped titanium target with a $300 \mathrm{keV}$ deuterium beam [7]. The neutron beam at $2.5 \mathrm{MeV}$ was produced by the primary beam impinging on the beam dump, containing deuterium atoms. For this reason, the flux of the $2.5 \mathrm{MeV}$ neutrons was weaker and less monochromatic than the flux of the $14.1 \mathrm{MeV}$ neutrons. The two samples of CLYC scintillators were placed at $1.25 \mathrm{~m}$ from the neutron source. The neutron generator is at $4.5 \mathrm{~m}$ from the floor and walls, to reduce the thermal neutron background. Both CLYC detectors were used for the measurement of $14.1 \mathrm{MeV}$ neutrons, whereas only CLYC-7 was used to detect neutrons at 2.5 $\mathrm{MeV}$ since the energy of the thermal-neutron peak is expected to overlap with the proton energy produced by the ${ }^{35} \mathrm{Cl}(\mathrm{n}, \mathrm{p})^{35} \mathrm{~S}$ reaction, because of the Q-value of the reaction, $0.6 \mathrm{MeV}$, to be added to the neutron energy. 
The left and right panels of figure 2 show the PSD matrices obtained with $14.1 \mathrm{MeV}$ neutrons measured in CLYC-6 and in CLYC-7 respectively. In both cases, the discrimination between gamma rays and neutrons is clearly visible. The thermal neutron peak is present only in the left panel of figure 2 at about 3.2 MeVee. In the figure, the neutron events show two components probably due to protons and $\alpha$ particles produced by the two reactions on ${ }^{35} \mathrm{Cl}$ as suggested in Ref [4]. The neutron contribution appears as a continuum without any distinct peak. This feature may be connected to the different twobody and three-body reaction mechanisms that may occur in these crystals with neutrons of $14.1 \mathrm{MeV}$.
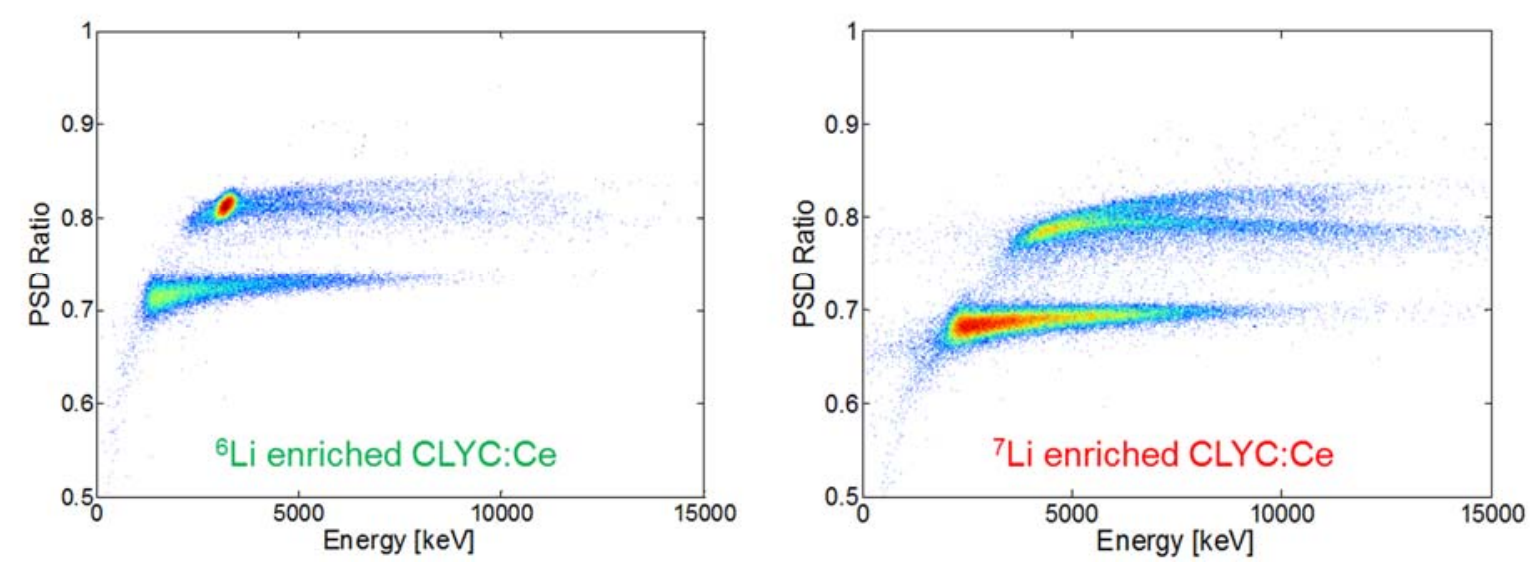

Fig. 2: the PDS matrixes obtained with fast neutrons of $14.1 \mathrm{MeV}$ with CLYC-6 (left panel) and CLYC-7 (right panel), respectively. The matrix obtained with CLYC-6 shows thermal-neutron peak. The peak is located at $\mathrm{x} \sim 3200$ and $\mathrm{y} \sim 0.8$. The $\mathrm{z}$ axis is in log scale.

Figure 3 shows the resulting PSD matrix at $2.5 \mathrm{MeV}$ measured with CLYC-7 scintillator. The fast neutron are highlighted by a red circle in the figure. The FWHM of the proton peak is dominated by the spread in the incident neutron energy. The additional time of flight measurement would allow the clean distinction between thermal and $2.5 \mathrm{MeV}$ neutrons (see section 3.2).

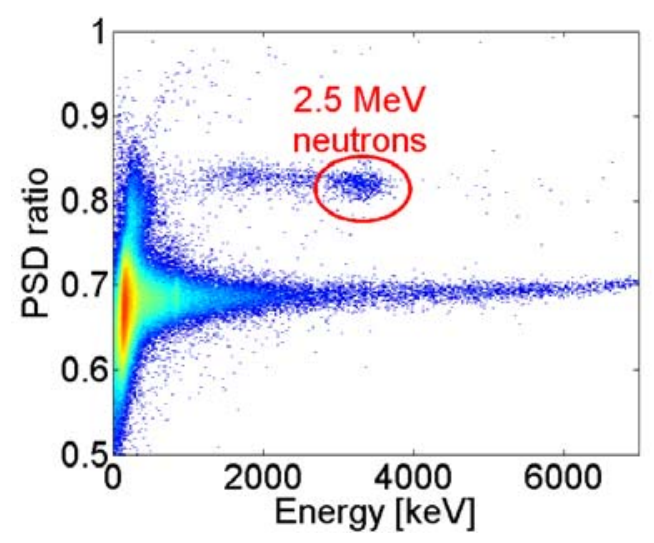

Fig. 3: The PDS matrix obtained with fast neutron of $2.5 \mathrm{MeV}$ measured with CLYC-7 scintillator. The neutron peak, related to reaction ${ }^{35} \mathrm{Cl}(\mathrm{n}, \mathrm{p})^{35} \mathrm{~S}$ is indicated by the circle. The $\mathrm{z}$ axis is in log scale.

\subsection{Fast Neutron Measurements at LNL}

The measurements were performed at LNL, on April 2015. The neutrons were produced making a proton beam impinging on a ${ }^{7} \mathrm{LiF}$ target. We selected different proton beam energies $(4.5-5-5.5 \mathrm{MeV})$ to produce neutrons with energies from $1.9 \mathrm{MeV}$ (detector at $90^{\circ}$ - proton beam energy $4.5 \mathrm{MeV}$ ) up to $3.8 \mathrm{MeV}$ (detector at $0^{\circ}$ - proton beam energy 5.5 MeV). Exploiting the RF time signal, it was also possible to measure the neutron kinetic energy with the TOF technique. 
The two cylindrical 1" $x$ 1" CLYC scintillators were coupled with HAMAMATSU R6233-100Sel photomultiplier tubes (PMT) and with two standard HAMAMATSU E1198-26 and E1198-27 voltage dividers. The detectors were placed at $0.7 \mathrm{~m}$ from the target for the TOF measurements and at $0^{\circ}$ and $90^{\circ}$, from the beam line, to measure of each proton beam energy two different neutrons energies. The anode signal was digitized with a 12 bit, $600 \mathrm{MHz}$ LeCroy waverunner HRO 66Zi oscilloscope (digital acquisition system). The energy spectra were obtained by integrating the signal for $3 \mu \mathrm{s}$. In the digital acquisition system, the neutron gamma discrimination was performed by integrating the signals within two different windows: the first one (W1) from the onset of the signal to $80 \mathrm{~ns}$, the second one (W2) from $100 \mathrm{~ns}$ to $600 \mathrm{~ns}$. The PSD ratio was defined as: $\mathrm{R}=\mathrm{W} 2 /(\mathrm{W} 2+\mathrm{W} 1)$ to be used to discriminate between gamma ray and neutrons. A digital CFD algorithm was used to provide the time spectra. The trigger of the experiment (for both the acquisition system) was the coincidence between the OR of the two CLYC detectors and the pulse beam.
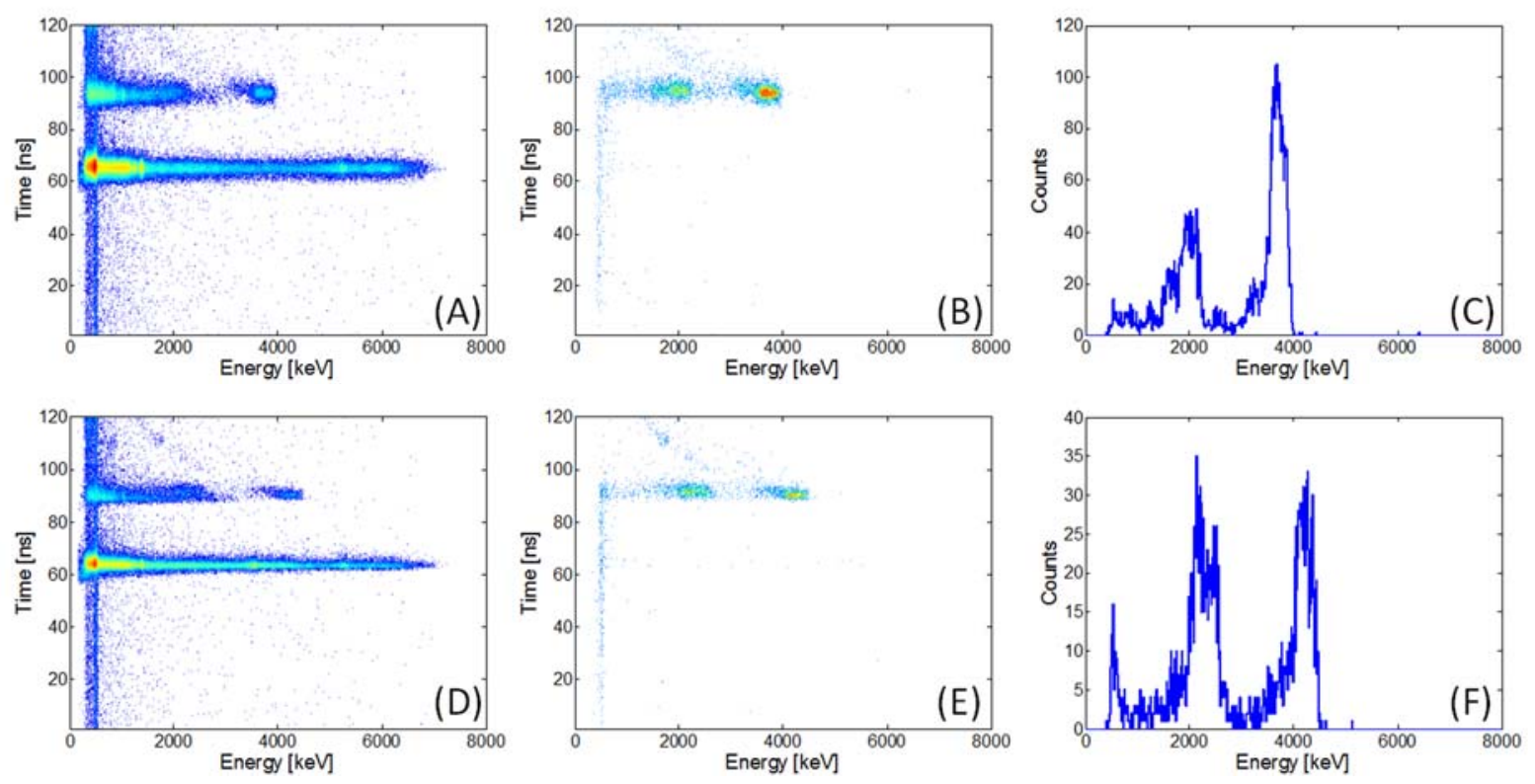

Fig. 4: Panel (A): The time vs energy matrix of the CLYC-7 scintillator. The proton beam energy was $5 \mathrm{MeV}$ and the detector was place at $0^{\circ}$. Panel (B): The same matrix of panel (A) with the condition that the events have to be discriminated as neutrons from the PSD. Panel (C): The measured energy spectrum of the neutrons. The neutron events were selected by the PSD and by the TOF. The two peaks of the reaction ${ }^{35} \mathrm{Cl}(\mathrm{n}, \mathrm{p}){ }^{35} \mathrm{~S}$ and ${ }^{35} \mathrm{Cl}(\mathrm{n}, \alpha){ }^{32} \mathrm{P}$ are visible. Panels (D), (E) and (F) are the same of panels (A), (B) and (C), the difference is the neutron energy. The $\mathrm{z}$ axis of the matrix is in $\log$ scale.

Figure 4 shows the preliminary results of the experiment, using the digital acquisition system. Only two of the six configuration (three proton energies and two detector angles) measured during the experiment are shown in figure 3. The results for CLYC-7 scintillator are reported. Panels (A) and (D) show the time vs energy matrix. The proton beam energy was 5 and $5.5 \mathrm{MeV}$ for top panels ((A)-(B)(C)) and for bottom panels ((D)-(E)-(F)), respectively. The detector was placed at $0^{\circ}$, therefore the neutrons are expected with an energy of 3.3 and $3.8 \mathrm{MeV}$ for panels top panels ((A)-(B)-(C)) and for bottom panels ((D)-(E)-(F)), respectively. Panels (B) and (E) show the same matrix of panels (A) and (D) selecting neutrons using PSD. The TOF difference between panel (B) and panel (E) is about $\sim 2 \mathrm{~ns}$. Panels $(\mathrm{C})$ and $(\mathrm{F})$ show the measured energy spectra of the neutrons. The neutron events was selected by the PSD and by the TOF. The two peak of the reaction ${ }^{35} \mathrm{Cl}(\mathrm{n}, \mathrm{p}){ }^{35} \mathrm{~S}$ and ${ }^{35} \mathrm{Cl}(\mathrm{n}, \alpha)^{32} \mathrm{P}$ are visible. The peak of ${ }^{35} \mathrm{Cl}(\mathrm{n}, \mathrm{p}){ }^{35} \mathrm{~S}$ of panel $(\mathrm{C})$ is at $\sim 3.9 \mathrm{MeV}(3.3 \mathrm{MeV}$ plus the $\mathrm{Q}$ value of $0.6 \mathrm{MeV})$, while the same peak of panel (F) is at $\sim 4.4 \mathrm{MeV}(3.8 \mathrm{MeV}$ plus the $\mathrm{Q}$ value of $0.6 \mathrm{MeV})$. 
Similar results was found for the CLYC-6 detector. The CLYC- 6 detects about 400 times more thermal neutrons than the CLYC-7. Therefore, the thermal neutrons are also detected, but they can be discriminated by the fast neutrons exploiting the TOF measurements.

Furthermore, the anode signal was formed with an amplifier (a modified BAFPRO unit [8]) with a shaping time of approximately $2 \mu$ s (slow component, the integral of the anode signal) and then was sent to an CAEN VME-ADC. The time spectra were obtained from the modified BAFPRO unit (shaping delay of $8 \mathrm{~ns}$ ) and a CAEN VME-TDC. The BAFPRO unit provides also a fast component, that is proportional to the maximum of the signal. The PSD, between gamma rays and neutrons, was performed producing a fast vs slow matrix. The data were acquires with a KMAX environment DAQ system [9] (analog acquisition system).

\section{Conclusions}

In this work, we presented the results from the response of two 1"x 1" samples of CLYC scintillators at fast and thermal neutrons: one enriched with $95 \%$ of ${ }^{6} \mathrm{Li}$ (CLYC-6) and the other with an enrichment of ${ }^{7}$ Li higher than 99\% (CLYC-7).

The thermal neutrons, produced by an AmBe source surrounded by paraffin, were measured with both detectors. It was observed that the thermal neutron detection efficiency for the CLYC-7 is $0.3 \%$ with respect to the CLYC-6 sample.

In order to test the capability of these crystals to detect fast neutrons, two experiments were performed one at the Frascati Neutron Generator facility (Italy) and the other one at LNL (Italy). At the neutron generator facility, a deuterium beam was used to provide neutrons of $2.5 \mathrm{MeV}$ or $14.1 \mathrm{MeV}$, respectively. We found a clear peak related to $2.5 \mathrm{MeV}$ neutrons while a continuum energy spectrum for $14.1 \mathrm{MeV}$ neutrons owing to a superimposition of different reaction mechanisms. Fast neutrons, from 1.9 MeV up to 3.8 MeV, were measured also at the CN accelerator of LNL. In this case, fast neutrons were identified by both the energy signal and the TOF technique. Exploiting the TOF technique, it is possible to subtract the thermal neutrons, especially for CLYC-6 detector.

\section{Acknowledgements}

The project is co-financed by the European Union and the European Social Fund. This work was also supported by NUPNET - ERA-NET within the the NuPNET GANAS project, under grant agreement $\mathrm{n}^{\circ}$ 202914 and from the European Union, within the " $7^{\text {th }}$ Framework Program" FP7/2007-2013, under grant agreement $n^{\circ} 262010$ - ENSAR-INDESYS. This work was also supported by "Programmi di Ricerca Scientifica di Rilevante Interesse Nazionale" (PRIN) number2001024324_01302.

\section{References}

[1] J. Glodo et al. Journal of Crystal Growth 379(2013)73-78.

[2] N. D’Olympia et al. Nucl. Instr. and Meth. A714(2013)121.

[3] Pointwise ENDF-VII library at $300 \mathrm{~K}$ : http://atom.kaeri.re.kr/cgi-bin/endfplot.pl

[4] N. D’Olympia at al., Nucl. Instr. and Meth, A763, (2014), 433.

[5] N. D’Olympia et al. Nucl. Instr. and Meth. A694(2012)140.

[6] M.B. Smith et al. IEEE TRANSACTIONS ON NUCLEAR SCIENCE, 60(2013)855.

[7] M. Martone, M.Angelone, M.Pillon.,Journal of Nuclear Materials 212 (1994) 1661.

[8] C.Boiano, et al, 2010 IEEE NSS and MIC Conference Record, 268.

[9] S.Brambilla, et al., 2012 IEEE NNS and MIC Conference Record, 1087. 